Supporting Information

\title{
Cancer-selective bioreductive chemotherapy mediated by dual hypoxia-responsive nanomedicine upon photodynamic therapy-induced hypoxia aggravation
}

Rongying Zhu ${ }^{\mathrm{a}, \#}$, Hua He ${ }^{\mathrm{b}, \#}$, Yong Liu ${ }^{\mathrm{c}, *}$, Desheng Cao ${ }^{\mathrm{b}}$, Jin Yan ${ }^{\mathrm{b}}$, Shanzhou Duan ${ }^{\mathrm{a}}$, Yongbing Chen ${ }^{\mathrm{a}, *}$, Lichen Yin ${ }^{\mathrm{b}, *}$

${ }^{a}$ Department of Thoracic Surgery, The Second Affiliated Hospital of Soochow University, Suzhou 215004, China

b Jiangsu Key Laboratory for Carbon-Based Functional Materials and Devices, Institute of Functional Nano \& Soft Materials (FUNSOM), Collaborative Innovation Center of Suzhou Nano Science \& Technology, Soochow University, Suzhou 215123, China

${ }^{\mathrm{c}}$ University of Groningen and University Medical Center Groningen, Department of Biomedical Engineering, Antonius Deusinglaan 1, 9713 AV, Groningen, The Netherlands

\# These authors contributed equally.

*Corresponding authors.

Email: chentongt@ sina.com (Y. Chen); Phone: 86-512-67784776

lcyin@suda.edu.cn (L. Yin); Phone: 86-512-65882039

y.liu@umcg.nl (Y. Liu); Phone: 31-06-39396957 


\section{Methods}

\section{Instrumentation}

${ }^{1} \mathrm{H}$ NMR spectra were recorded on a Varian U400 $\mathrm{MHz}$ spectrometer. Morphology of the NPs was observed by transmission electron microscopy (TEM, Tecnai G220). Size of the NPs was determined by dynamic laser scanning (DLS, Malvern Zetasizer). UV-Vis spectrum was collected on a UV-Vis spectrophotometer (Genesys 10s, Thermo Scientific). Flow cytometry analysis was performed on a BD FACS Calibur flow cytometer (Beckton Dickinson, USA). The oxygen levels in the tumors were detected using a photoacoustic imaging system (FujiFilm VisualSonics Inc.) in the Oxy-hem mode (750 and $850 \mathrm{~nm}$ for deoxygenated and oxygenated hemoglobins, respectively). The oxyhemoglobin saturation was represented by PA signal ratios between oxygenated and total hemoglobins.

\section{Cytotoxicity of NPs}

To evaluate the cytotoxicity of the nanocarrier, 4T1 cells were seeded on 96-well plates at $5 \times 10^{3}$ cells/well and cultured for $24 \mathrm{~h}$, followed by addition of PA/HA-Ce6 NPs at various final concentrations. Cells were incubated for $72 \mathrm{~h}$ before viability assessment by the MTT assay.

\section{Pharmacokinetics and biodistribution}

1,1-Dioctadecyl-3,3,3,3-tetramethylindotricabocyanine iodide (DiR) as a fluorescent dye was encapsulated into the NPs. For the pharmacokinetics study, female BALB/c mice were i.v. injected with PA/HA@DiR NPs, or free DiR at 1.25 $\mathrm{mg} \mathrm{DiR} / \mathrm{kg}$. Blood $(20 \mu \mathrm{L})$ was collected from the orbit at different time points post injection, and was dissolved in lysis buffer (1\% Triton X-100, $100 \mu \mathrm{L})$ with sonication. DIR was extracted via incubation of the blood samples in HCl-IPA at $-20{ }^{\circ} \mathrm{C}$ overnight. After centrifugation at $12000 \mathrm{rpm}$ for $30 \mathrm{~min}$, DiR level in the supernatant was determined by spectrofluorimetry $\left(\lambda_{\mathrm{ex}}=748 \mathrm{~nm}, \lambda_{\mathrm{em}}=780 \mathrm{~nm}\right)$.

For the biodistribution study, female BALB/c mice were unhaired, anesthetized with isoflurane, and subcutaneously injected with $4 \mathrm{~T} 1$ cells $\left(2 \times 10^{6}\right)$ at the left mammary pad. When the tumor volume reached $150 \mathrm{~mm}^{3}$, mice were i.v. injected with PA/HA@DiR NPs or free DiR at 1.25 mg DiR/kg. Fluorescence imaging of anesthetized animals was performed at various time points post i.v. injection using an 
in vivo imaging system (IVIS® Lumina III, PerkinElmer, USA). In a parallel study, at $6 \mathrm{~h}$ post i.v. injection, mice were sacrificed. The tumor tissues and major organs including heart, liver, spleen, lung, and kidney were collected, washed with PBS, and subjected to fluorescence imaging.

\section{Acute toxicity evaluation}

PA/HA-Ce6@TPZ NPs or PBS were i.v. injected to normal BALB/c mice at 2 $\mathrm{mg}$ TPZ/kg. At $24 \mathrm{~h}$ injection, blood was collected, and the hematological assessment was performed on a BC-5380 automatic chemistry analyzer (Mindray, China) including white blood cell count (WBC), red blood cell count (RBC), hemoglobin (HGB), hematocrit, and platelet count (PLT), The blood was centrifuged at 12000 rpm for $10 \mathrm{~min}$, and the supernatant was subjected to biochemical parameter assessment on a Cobas501 automatic hematology analyzer (Roche, U.S.A.), including alanine transaminase (ALT), aspartate transaminase (AST), total protein (TP), albumin (ALB), blood urea nitrogen (BUN), and creatinine (CREA).

\section{Statistical analysis}

Data were represented as the average \pm standard deviation. Significance comparison was performed using a Student's t-test or analysis of variance (ANOVA) coupled with Tukey's post-hoc analysis. 


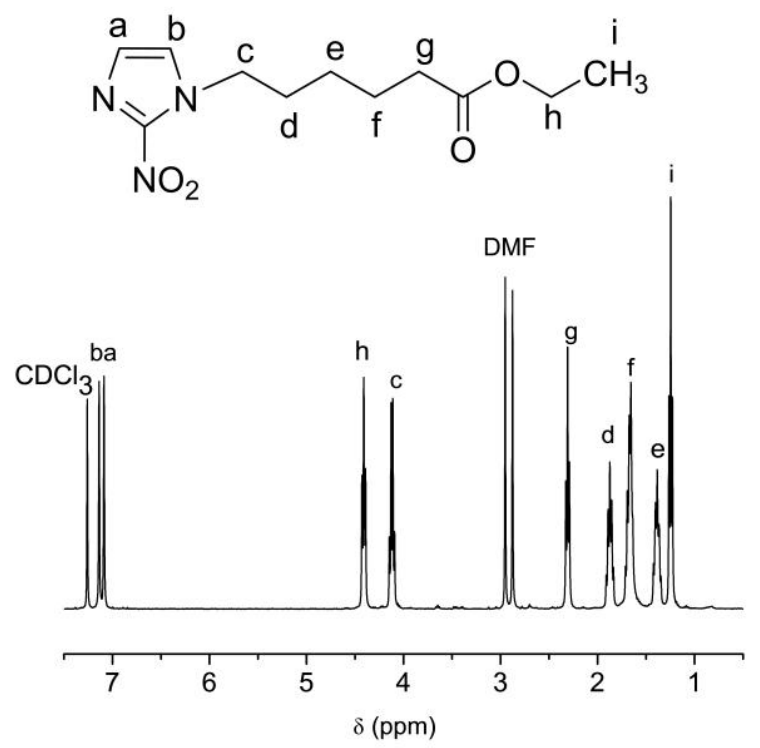

Figure S1. ${ }^{1} \mathrm{H}$ NMR spectrum of compound $1\left(\mathrm{CDCl}_{3}, 400 \mathrm{MHz}\right)$. 


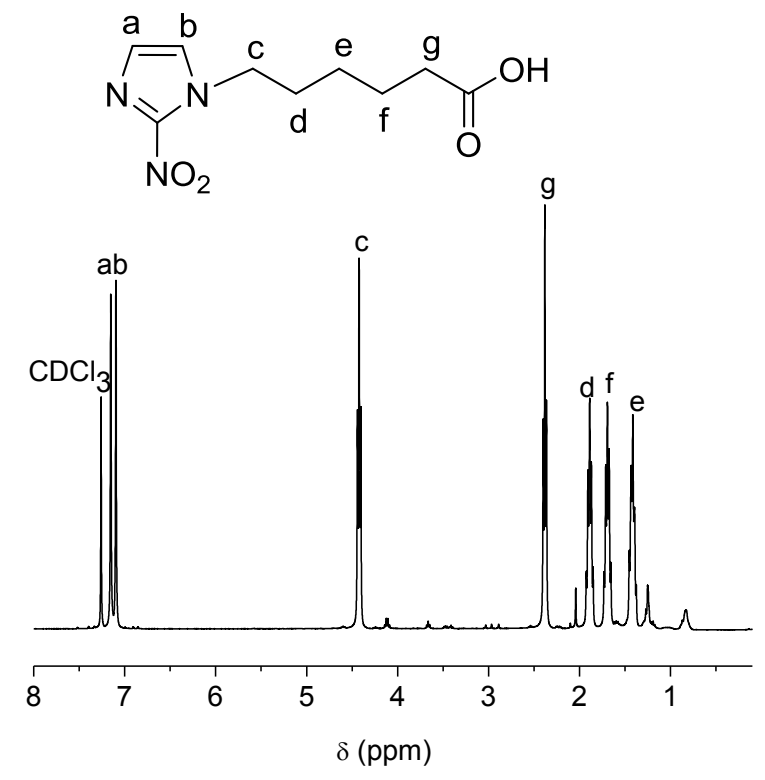

Figure S2. ${ }^{1} \mathrm{H}$ NMR spectrum of compound $2\left(\mathrm{CDCl}_{3}, 400 \mathrm{MHz}\right)$. 


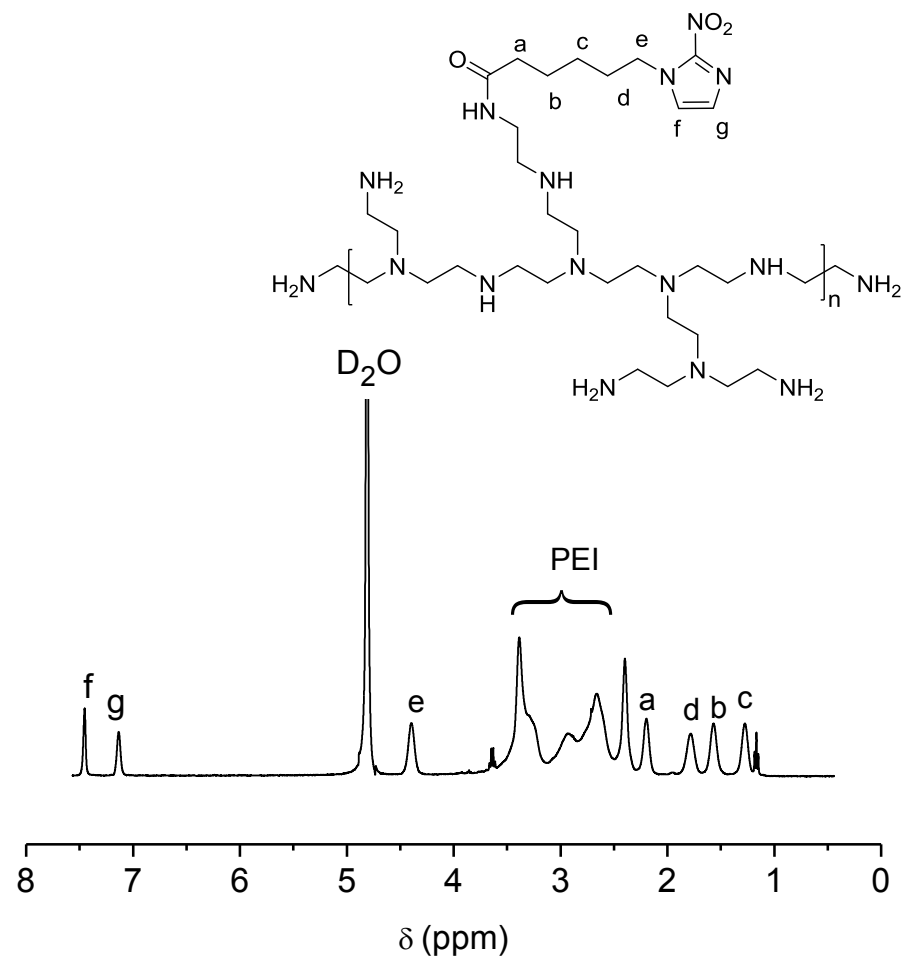

Figure S3. ${ }^{1} \mathrm{H}$ NMR spectrum of $\mathrm{PA}\left(\mathrm{D}_{2} \mathrm{O}, 400 \mathrm{MHz}\right)$. 


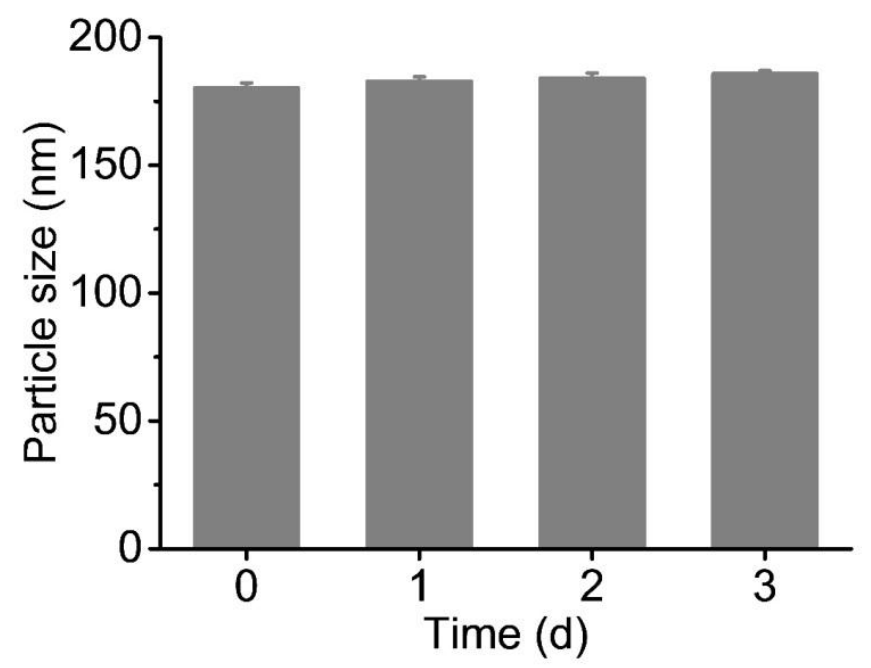

Figure S4. Size alteration of PA/HA-Ce6@TPZ NPs NPs following incubation in 10\% FBS for up to 3 days. 


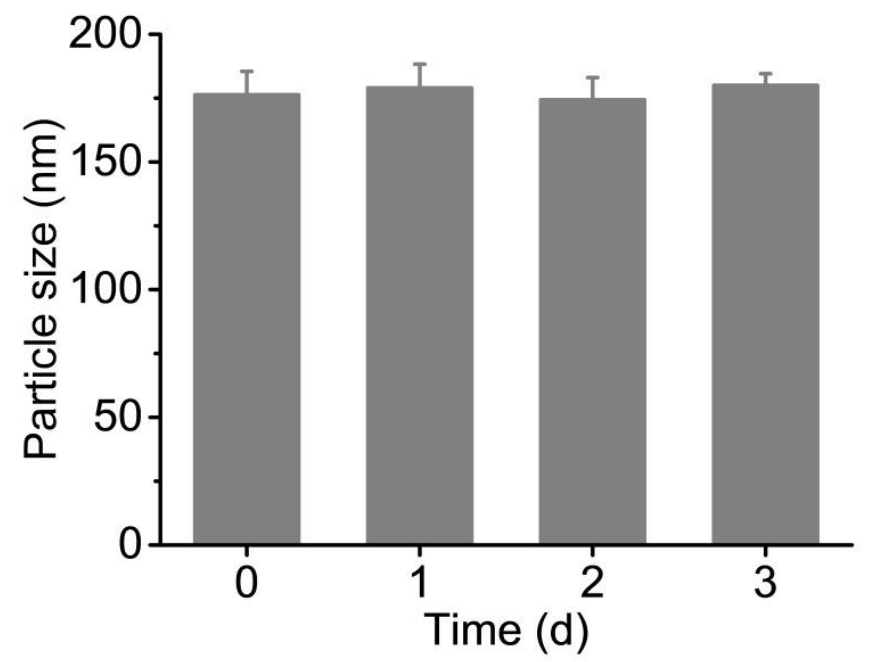

Figure S5. Size alteration of PA/HA-Ce6@TPZ NPs NPs following incubation in normal saline for up to 3 days. 


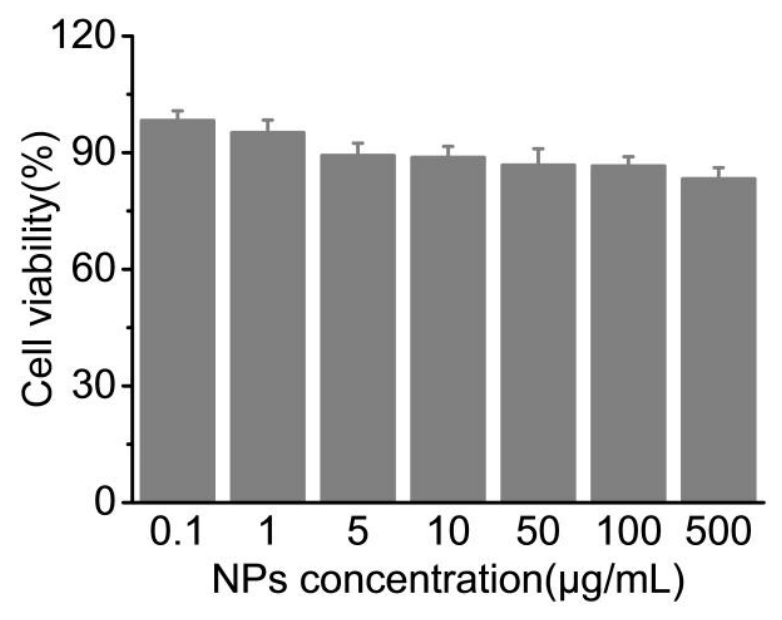

Figure S6. Cytotoxicity of PA/HA-Ce6 NPs in 4T1 cells at various final concentrations following incubation for $72 \mathrm{~h}(\mathrm{n}=3)$. 


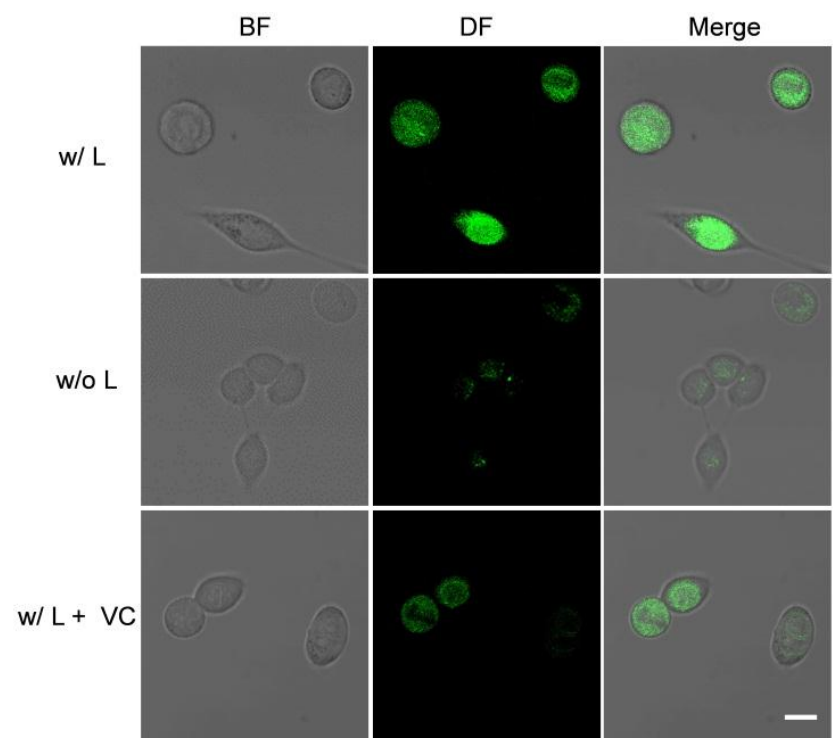

Figure S7. Intracellular ROS generation in 4T1 cells incubated with PA/HA-Ce6@TPZ NPs for $4 \mathrm{~h}$ and irradiated $\left(660 \mathrm{~nm}, 2 \mathrm{mw} / \mathrm{cm}^{2}\right)$ for $30 \mathrm{~min}$ as detected via DCFH-DA staining. Cells were pre-treated VC $(200 \mu \mathrm{M})$ for $4 \mathrm{~h}$ and during the 4-h incubation time to deplete ROS generation. Bar represents $10 \mu \mathrm{m}$. 


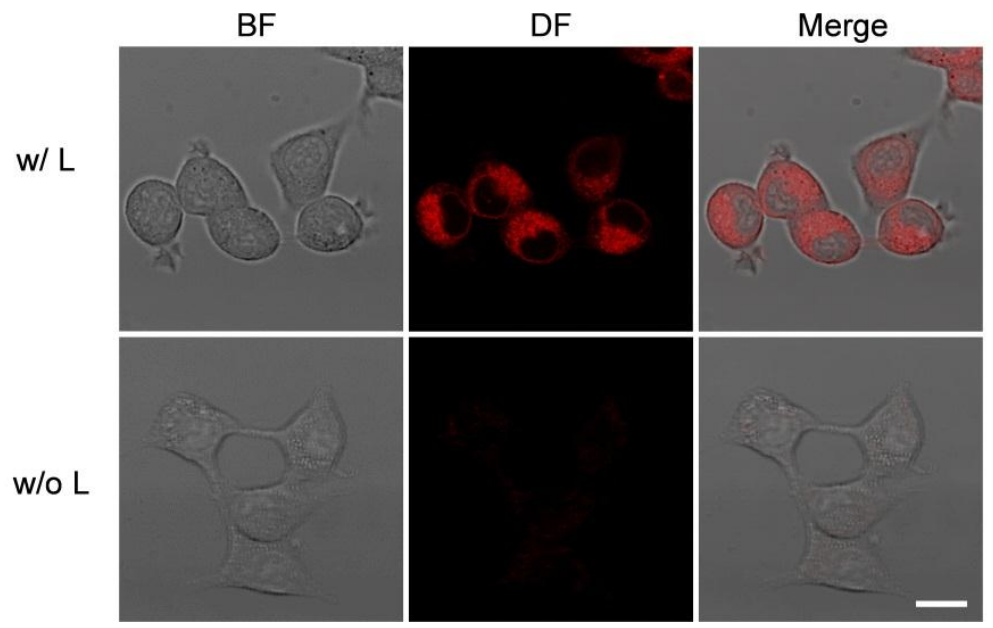

Figure S8. Intracellular hypoxia levels in 4T1 cells incubated with PA/HA-Ce6@TPZ NPs for $4 \mathrm{~h}$ and irradiated $\left(660 \mathrm{~nm}, 2 \mathrm{mw} / \mathrm{cm}^{2}\right)$ for $30 \mathrm{~min}$ as detected using the Hypoxia/Oxidative Stress Detection Kit. Bar represents $10 \mu \mathrm{m}$. 


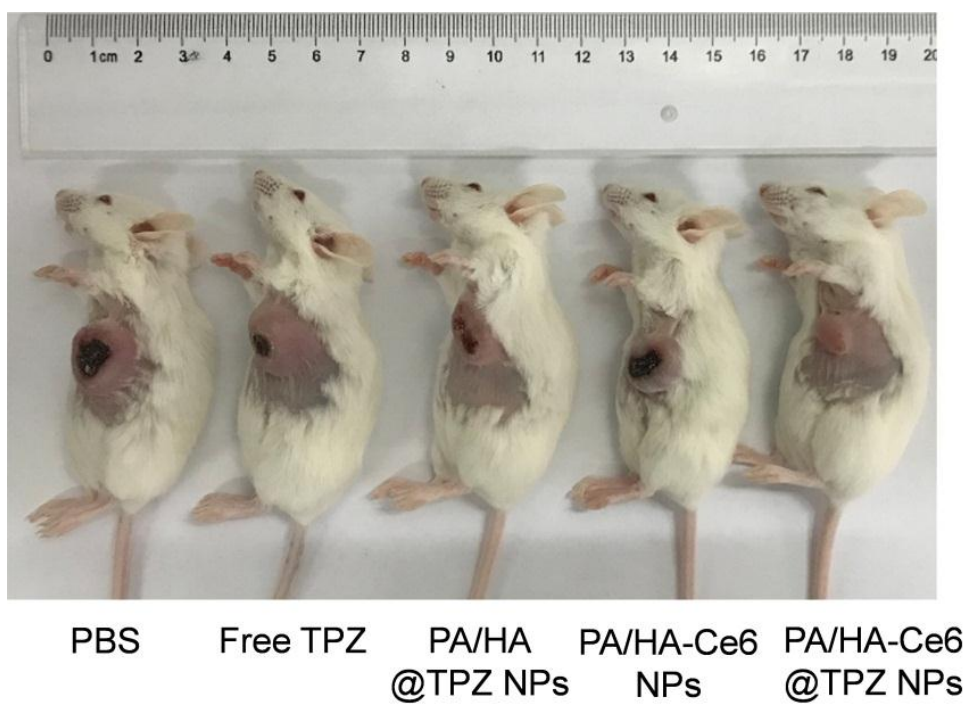

Figure S9. Representative 4T1 tumor-bearing mice on day 12 in the in vivo efficacy study. 


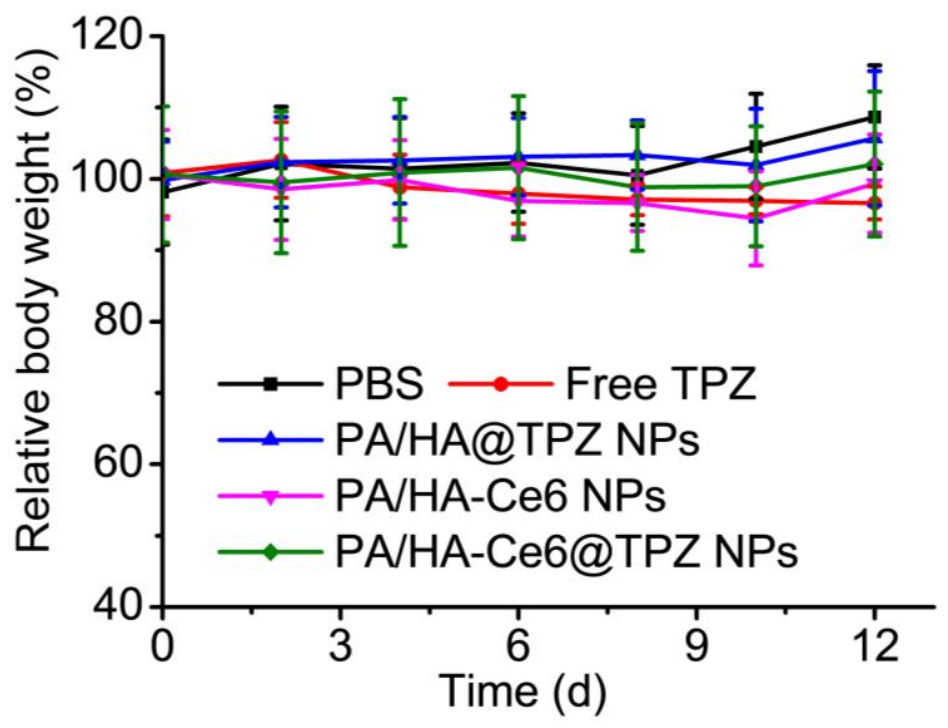

Figure S10. Body weight of 4T1 tumor-bearing mice within the 12-day observation period in the in vivo efficacy study $(\mathrm{n}=8)$. 


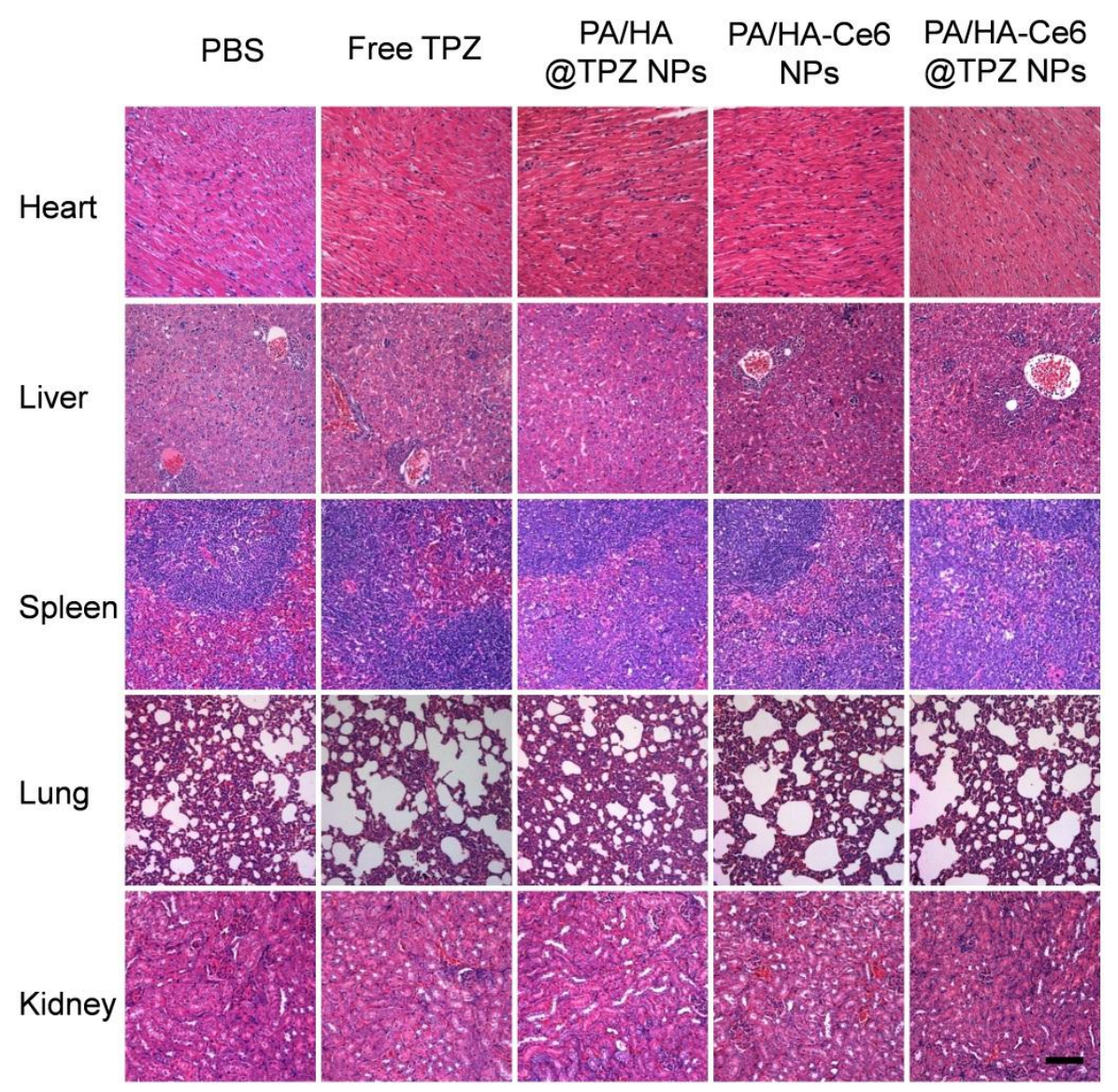

Figure S11. H\&E staining of major organ sections harvested from 4T1 tumor-bearing mice on day 12 in the in vivo anti-tumor efficacy study. Bar $=100 \mu \mathrm{m}$. 

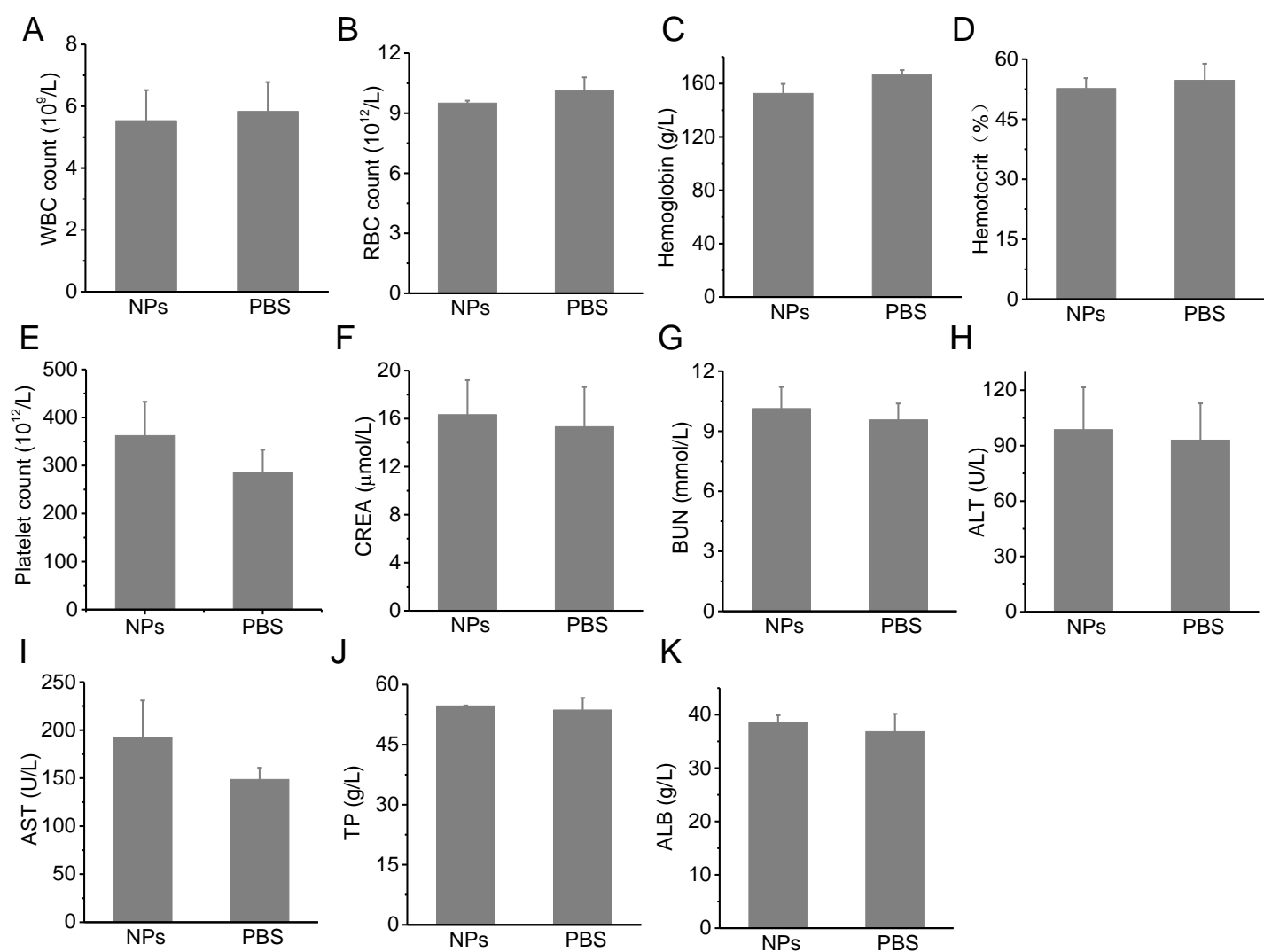

Figure S12. Hematological and biochemical analysis after i.v. injection of PA/HA-Ce6@TPZ NPs (2 mg TPZ/kg) and PBS ( $=3)$. 